\title{
Kinematic analysis of imaging seekers with roll-over-nod gimbal and a folded electro-optical layout (Erratum)
}

Kutlu Kandemir, Yigit Yazicioglu, Bulent Ozkan

Kutlu D. Kandemir, Yigit Yazicioglu, Bulent Ozkan, "Kinematic analysis of imaging seekers with roll-over-nod gimbal and a folded electro-optical layout (Erratum)," Proc. SPIE 11159, Electro-Optical and Infrared Systems: Technology and Applications XVI, 1115912 (20 February 2020); doi: $10.1117 / 12.2569832$

SPIE. Event: SPIE Security + Defence, 2019, Strasbourg, France 


\section{Kinematic analysis of imaging seekers with roll-over-nod gimbal and a folded electro-optical layout (Erratum)}

Kutlu D. Kandemir, ${ }^{1}$ Yigit Yazicioglu, ${ }^{2}$ Bulent Ozkan ${ }^{3}$

${ }^{1}$ ASELSAN A.S. (Turkey), ${ }^{2}$ Middle East Technical Univ. (Turkey), ${ }^{3}$ TÜBITAK SAGE (Turkey)

Proceedings Volume 11159, Electro-Optical and Infrared Systems: Technology and Applications

$\underline{\mathrm{XVI}}$; 111590S (2019) https://doi.org/10.1117/12.2532791

Event: SPIE Security + Defence, 2019, Strasbourg, France

Online Publication Date: 9 October 2019

Erratum Published: 20 February 2020

A revised version of this manuscript was published on 20 February 2020. Details of the revision are provided in the text that accompanies this Erratum. The original paper has been updated.

Revisions to Equations (28) and (29)

Original

$$
\begin{aligned}
& \mathrm{D}_{\mathrm{s}}\left(\overrightarrow{\mathrm{r}}_{\mathrm{t} / \mathrm{s}}\right)+\vec{\omega}_{\mathrm{s} / \mathrm{o}} \times \overrightarrow{\mathrm{r}}_{\mathrm{t} / \mathrm{s}}=\overrightarrow{\mathrm{V}}_{\mathrm{t}}-\overrightarrow{\mathrm{V}}_{\mathrm{m}}-\overrightarrow{\mathrm{\omega}}_{\mathrm{m} / \mathrm{o}} \times \overrightarrow{\mathrm{r}}_{\mathrm{s} / \mathrm{m}} \\
& \mathrm{D}_{\mathrm{s}}\left(\overrightarrow{\mathrm{r}}_{\mathrm{t} / \mathrm{s}}\right)+\vec{\omega}_{\mathrm{s} / \mathrm{o}} \times \overrightarrow{\mathrm{r}}_{\mathrm{t} / \mathrm{s}}=\overrightarrow{\mathrm{V}}_{\mathrm{t}}-\overrightarrow{\mathrm{V}}_{\mathrm{m}}-\overrightarrow{\mathrm{\omega}}_{\mathrm{m} / \mathrm{o}} \times \overrightarrow{\mathrm{r}}_{\mathrm{s} / \mathrm{m}}
\end{aligned}
$$

$\underline{\text { New }}$

$$
\begin{aligned}
& \left(\vec{\omega}_{s / m}+\vec{\omega}_{m / o}\right) \times \vec{r}_{t / s}=\vec{V}_{t}-\vec{V}_{m}-\vec{\omega}_{m / 0} \times d \vec{u}_{l}^{(m)}-D_{s}\left(\vec{r}_{t / s}\right) \\
& \vec{\omega}_{s / m} \times \vec{r}_{t / s}=\vec{V}_{t}-\vec{V}_{m}-D_{s}\left(\vec{r}_{t / s}\right)-\vec{\omega}_{m / 0} \times d \vec{u}_{l}^{(m)}-\vec{\omega}_{m / o} \times{\overrightarrow{r_{t} / s}}^{(m)}
\end{aligned}
$$

\section{Revisions to Equation (37)}

Original

$$
\mathrm{F} \frac{\mathrm{d}}{\mathrm{dt}}\left(\overline{\mathrm{r}}_{v / \mathrm{s}}{ }^{(0)}\right)=\left[\begin{array}{c}
-\mathrm{c}(\psi) \mathrm{s}(\theta) \dot{\psi}-\mathrm{c}(\psi) \mathrm{s}(\theta) \dot{\theta} \\
\mathrm{c}(\psi) \mathrm{c}(\theta) \dot{\psi}-\mathrm{s}(\psi) \mathrm{s}(\theta) \dot{\theta} \\
-\mathrm{c}(\theta) \dot{\theta}
\end{array}\right]=\left[\begin{array}{l}
\mathrm{t}_{1} \\
\mathrm{t}_{2} \\
\mathrm{t}_{3}
\end{array}\right]=\left[\begin{array}{c}
-\mathrm{s}(\eta) \dot{\eta} \\
\mathrm{c}(\eta) \mathrm{s}(\rho) \dot{\eta}+\mathrm{c}(\rho) \mathrm{s}(\eta) \dot{\rho} \\
-\mathrm{c}(\eta) \mathrm{c}(\rho) \dot{\eta}+\mathrm{s}(\eta) \mathrm{s}(\rho) \dot{\rho}
\end{array}\right]
$$

New

$$
F \frac{d}{d t}\left(\bar{r}_{t / s}(\theta)\right)=\left[\begin{array}{c}
-s(\psi) c(\theta) \dot{\psi}-c(\psi) s(\theta) \dot{\theta} \\
c(\psi) c(\theta) \dot{\psi}-s(\psi) s(\theta) \dot{\theta} \\
-c(\theta) \dot{\theta}
\end{array}\right]=\left[\begin{array}{l}
t_{1} \\
t_{2} \\
t_{3}
\end{array}\right]=\left[\begin{array}{c}
-s(\eta) \dot{\eta} \\
c(\eta) s(\rho) \dot{\eta}+c(\rho) s(\eta) \dot{\rho} \\
-c(\eta) c(\rho) \dot{\eta}+s(\eta) s(\rho) \dot{\rho}
\end{array}\right]
$$

Electro-Optical and Infrared Systems: Technology and Applications XVI, edited by Duncan L. Hickman, Helge Bürsing, Proc. of SPIE Vol. 11159, 1115912 · C 2019 SPIE · CCC code: 0277-786X/19/\$21 · doi: 10.1117/12.2569832 